\title{
EFFECT OF INTERFACIAL PDNI CONCENTRATION ON TIME RESPONSE OF SI- BASED ELECTRODEPOSITED HYDROGEN SENSORS
}

\author{
L.Dong $^{1 *}$, C.H. de Groot ${ }^{1}$, A.Usgaocar ${ }^{1}$, and V. Chavagnac ${ }^{2}$ \\ ${ }^{1}$ Electronics and Computer Science, University of Southampton, Southampton, U.K \\ ${ }^{2}$ Géosciences Environnement Toulouse (GET) CNRS,UPS, Toulouse, France
}

\begin{abstract}
This paper presents Hydrogen sensors based upon electrodeposited PdNi-Si Schottky barriers which have been fabricated with a gradient structure in the PdNi concentration in a single electrodeposition run through variation of the deposition potential. The resulting sensors in the back to back diode configuration show very low idle leakage current and good sensitivity to hydrogen. The structure with increased $\mathrm{Ni}$ concentration at the PdNi-Si interface shows dramatically improved time response as compared to the uniform concentration sample. I-V and $\mathrm{C}-\mathrm{V}$ characteristics of these hydrogen sensors also depicts in this paper to determine the Schottky barrier height and ideality factor.
\end{abstract}

\section{KEYWORDS}

Schottky barrier; low power consumption; variable structures; fast response

\section{INTRODUCTION}

Hydrogen is considered as a clean combustible and renewable alternative to carbon-based fuels. Nowadays hydrogen has a rapidly increasing commitment of the utilization in international energy market [1].

Hydrogen sensors are crucial to enhancing safety in Hydrogen production, storage, transport and use [2] due to hydrogen gas having a number of combustion characteristics being, low minimum ignition energy $(0.015 \mathrm{~mJ})$, high heat of combustion $\left(147 \mathrm{~kJ} / \mathrm{g} \mathrm{H} \mathrm{H}_{2}\right)$, as well as a wide flammable range. Hydrogen is also impossible to be detected by human sense system on account of its lack of colour, odour and taste. Leak detection and monitoring is vital in Hydrogen applications especially as Hydrogen can embrittle storage containers and cause explosive leaks. The detection and monitoring for hydrogen has a history of nearly 100 years since the hydrogen measurements at filling stations for airship. Currently, there exists a broad range of hydrogen sensor options and technologies, and selecting an appropriate sensor technology can be compromised.

Solid state hydrogen sensors are of interest due to their simplicity and their compatibility with allied electronic systems. The import noble metals such as Pd or $\mathrm{Pt}$ were employed to catalyse the dissociation of Hydrogen molecules into atoms, and then the atoms diffuse into the silicon leading to a physical and electrical modification. The sensors based on resistivity change in $\mathrm{Pd} / \mathrm{Pd}$-alloy films draw currents in the microampere to milliampere range, and are therefore not very power efficient. Those that sense current changes in $\mathrm{Pt} / \mathrm{Pd}-$ semiconductor Schottky barriers consume significantly lower power.

In this paper, we present a method to speed up the response time through growth of a gradient in $\mathrm{PdNi}$ concentration with depth. We have previously introduced a hydrogen sensor based on electrodeposited PdNi-Si Schottky barriers [3]. The sensor is small in size and has an extremely low idle current due to its back to back diode configuration (Fig 1). The $\mathrm{Pd}$ and $\mathrm{Ni}$ were codeposited from the solution to create an alloy with $30 \%$ $\mathrm{Ni}$ which suppresses an irreversible phase change at higher Hydrogen concentrations that occurs in pure Pd. The sensors exhibit large change (up to a factor of 100) in current on exposure to gaseous Hydrogen. The sole significant drawback of the sensor was the slow response time of the order of minutes.

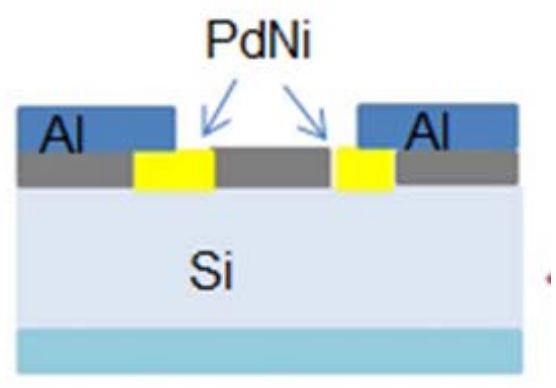

Figure 1: Cross section of a back to back PdNi-Si Schottky barrier Hydrogen sensor [5].

\section{FABRICATION}

We have previously introduced a hydrogen sensor based on electrodeposited PdNi-Si Schottky barriers [3]. The Hydrogen sensor was fabricated on a 1.0-2.0 $\Omega . \mathrm{cm}$, $<100>$ n-type silicon wafer. Low resistivity of the wafer is benefit for electrodeposition process. A lower resistivity gives a higher reverse bias, as shown in Fig 2, which leads to electrons tunnelling through the Schottky barrier. This reduces the sensitivity of the sensor to hydrogen as the reduction of the Schottky barrier height due to hydrogen gives rise to thermionic emission. High resistivity silicon is difficult to use for electrodeposition of PdNi films due to the ohmic voltage drop in the $\mathrm{Si}$ wafers. The chosen resistivity of a few $\Omega . \mathrm{cm}$ is a good compromise to fabricate Schottky barrier contacts for sensors. 


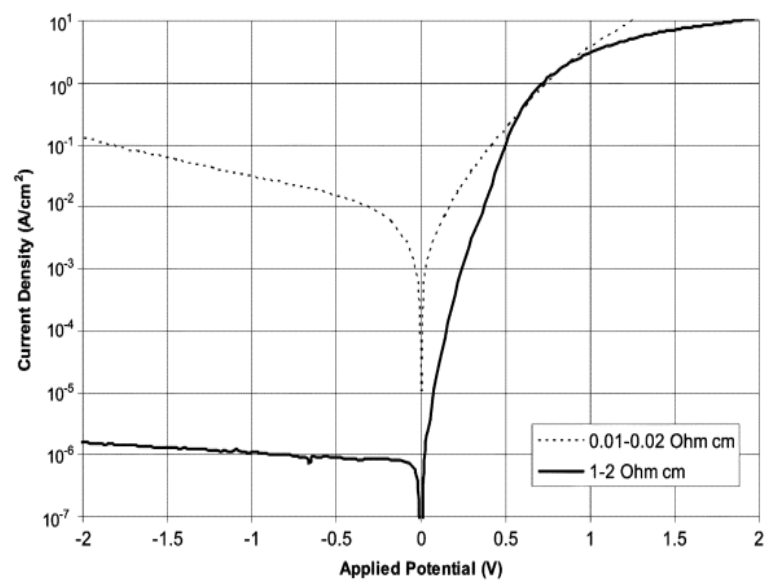

Figure 2: J-V curves of electrodeposited Ni-Si contacts for 1-2 S.cm and 0.01-0.02 S.cm [4]

The process flow is shown in Fig 3. In order to develop a homogeneous and clean thermal oxide on the Silicon surface, the sruface was initially rinsed in fuming nitride to remove organic residues, followed by dehydration and transfer the wafer to furnace for $50 \mathrm{~nm}$ thermal oxide growth, which act as the insulating layer for the second metal layer in the device. Next a 300nm layer of Aluminium was deposited on the back side of wafer by using evaporator. The oxide layer was then patterned by photolithography and the developed expose layer was etched away by 20:1 Hydrofluoric acid. In the following the wafer was immersed in the PdNi electrochemical solution and a 50nm PdNi film will be deposited into the area that was etched away in the previous step. The PdNi film on the exposed Silicon surface will form the Schottky diode. A second photolithography was utilised to pattern the substrate for contact pads and wires, these wires and pads were fabricated with $100 \mathrm{~nm} \mathrm{Al}$ by evaporating. The redundant $\mathrm{Al}$ on the Silicon wafer was lifted off by Acetone and the sample cleaned with Acetone and IPA.

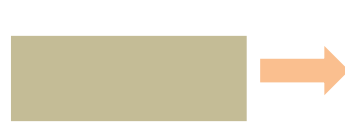

(A) $\mathrm{Si}$

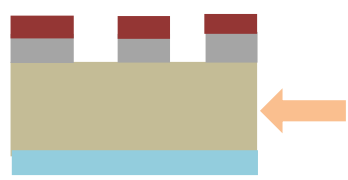

(D) Photolithography

(C) 300nm backside Al evaporation

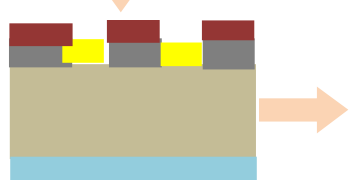

(E) PdNi Electrodeposition
(G) 100nm Al Evaporation On the top

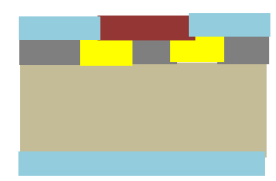

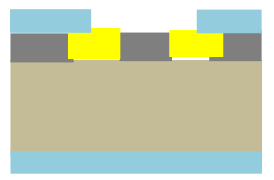

(H) Al Lift off
Figure 3: Fabrication Process of back to back Schottky barrier Hydrogen sensor [5].

The Pd and Ni were co-deposited from the solution to create an alloy with $30 \% \mathrm{Ni}$ which suppresses an irreversible phase change at higher Hydrogen concentrations that occurs in pure $\mathrm{Pd}$. The sensors exhibit large change (factor of 100) in current on exposure to gaseous Hydrogen. Despite this large change, the current was observed to be less than $500 \mathrm{nA}$ over the measured Hydrogen pressure range of 25 mbar. The sole significant drawback of the sensor was the slow response time of the order of 10 s of minute.

Fig 4 shows that a wide range of $\mathrm{Ni}$ concentrations can be achieved from a PdNi solution simply by varying the deposition potentials. The exactly atomic number for $\mathrm{Ni}$ and $\mathrm{Pd}$ are a function of potential. The concentration ratios of the elements are determined by the electrodeposition potential with larger negative voltages leading to high Pd concentration.

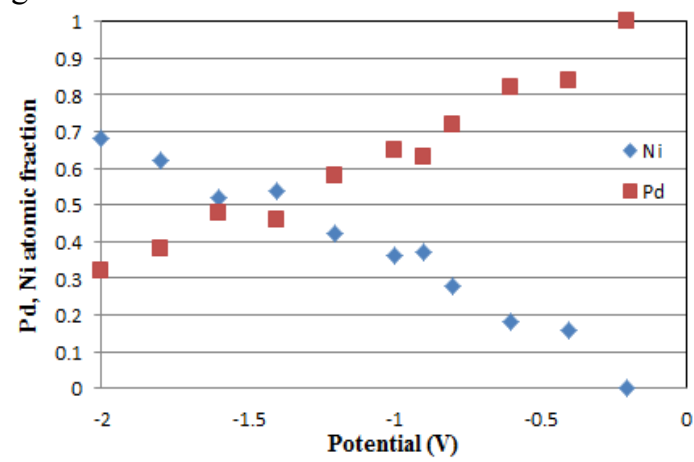

Figure 4: Energy dispersive $x$-ray analysis of $\mathrm{Pd}$ and $\mathrm{Ni}$ atomic fraction in films electro-deposited at different potentials from a single PdNi solution [5].

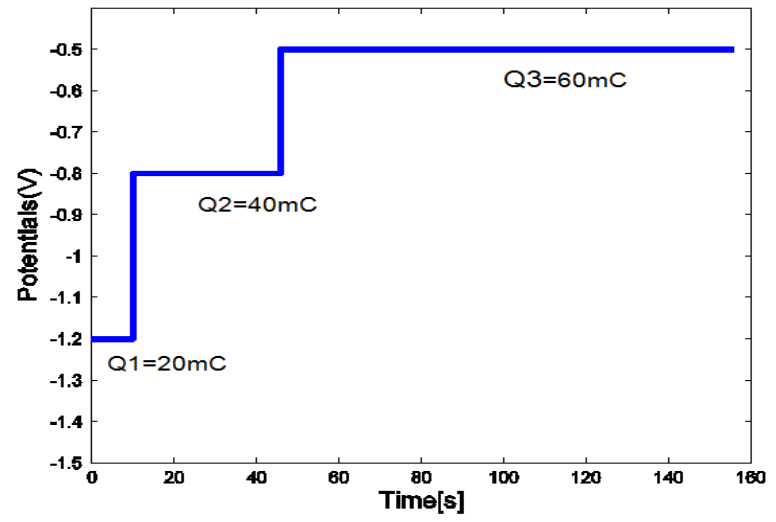

Figure 5: The potential variation during the electrodeposition with three steps. Each step corresponds to $20 m$ C of charge [5]. 
Three structures were made as shown in Fig 6. Structure A has uniform concentration of $30 \% \mathrm{Ni}$ concentration $\left(\mathrm{Pd}_{0.7} N i_{0.3}\right)$, structure $\mathrm{B}$ has an increased $\mathrm{Pd}$ concentration at the top surface to speed up $\mathrm{H} 2$ dissociation $(10 \% \mathrm{Ni}$ on the top of film) and structure $\mathrm{C}$ has as well an increased $\mathrm{Ni}$ concentration at the $\mathrm{Si}$ interface to improve the interface ion trapping $(40 \% \mathrm{Ni}$ on the bottom of the film).

\begin{tabular}{|c|c||c|}
\hline $30 \% \mathrm{Ni}$ & $10 \% \mathrm{Ni}$ & $10 \% \mathrm{Ni}$ \\
\hline $30 \% \mathrm{Ni}$ & $30 \% \mathrm{Ni}$ & $30 \% \mathrm{Ni}$ \\
\hline $30 \% \mathrm{Ni}$ & $30 \% \mathrm{Ni}$ & $40 \% \mathrm{Ni}$ \\
\hline $\mathrm{Si}$ & $\mathrm{Si}$ & $\mathrm{Si}$ \\
\hline
\end{tabular}

(A)

(B)

(C)

Figure 6: Schematic of electro-deposited PdNi Schottky barrier: (A) uniform concentration, (B) increased $P d$ concentration at the top surface, (C) increased $\mathrm{Ni}$ concentration at the Si interface [5].

When $-0.8 \mathrm{~V}$ was applied for the electrochemical deposition as seen from the EDX data the alloy film contains nearly $30 \% \mathrm{Ni}$ (Fig 5(A)). The higher the deposition potential applied the lower $\mathrm{Ni}$ concentration will be achieved and vice versa. The PdNi-Si Schottky barriers have been fabricated with a gradient structure in the PdNi concentration in a single electrodeposition run through variation of the deposition potential as the Fig 5 (a) shows. The total deposition process was divided into three steps for the $50 \mathrm{~nm} \mathrm{PdNi}$ structure variation. Initially, the electrodeposition potential is set-up on $-1.2 \mathrm{~V}$ for $40 \% \mathrm{Ni}$ concentration, the bottom layer of PdNi alloy film was formed after 7 seconds and the charge during the deposition reached $20 \mathrm{mC}$. The potential was then set to $0.8 \mathrm{~V}$ for $30 \% \mathrm{Ni}$ on the middle layer film. When the total charge reaches $40 \mathrm{mC}$ this process will be terminated and the potential will be decreased to $-0.5 \mathrm{~V}$ for $10 \% \mathrm{Ni}$ on the top layer of the film. The final process ends when the total reaches $60 \mathrm{mC}$, corresponding to the thickness PdNi film of $50 \mathrm{~nm}$.

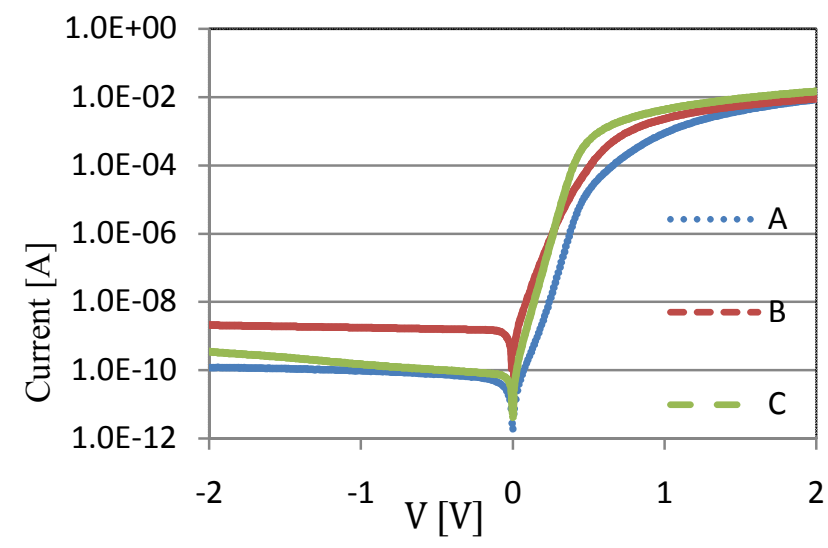

Figure 7: Current (I)-Voltage (V) characteristics of PdNiSi Schottky barrier hydrogen sensors with structures as described in Figure 6.
Fig7 illustrates the dependence of current on applied voltage of the three structures (A, B, C) Hydrogen sensor, which were grown on the $n$ type silicon wafer by $\mathrm{PdNi}$ electrodeposition. Table 1 lists the key parameters of these hydrogen sensor Schottky barriers. The Schottky barrier characteristics were analysed using the thermionic emission model. A linear fit of the low forward bias region of the I-V plot was show in the Fig 7 and the barrier height, ideality factor $\eta$ were listed in table 1 .

Table 1: The I-V characteristic parameters of three structures Schottky barrier hydrogen sensor, the Schottky contacts were made in the medium resistivity silicon wafer

\begin{tabular}{|l|c|c|c|c|}
\hline Structures & $\begin{array}{c}I_{S} \\
(\mathrm{~A})\end{array}$ & $\begin{array}{c}J_{S} \\
\left(\mathrm{~A} / \mathrm{cm}^{2}\right)\end{array}$ & $\begin{array}{c}\varphi_{B n} \\
(\mathrm{~V})\end{array}$ & $\eta$ \\
\hline $\mathrm{A}$ & $1.14 \times 10^{11}$ & $1.14 \times 10^{-9}$ & 0.92 & 1.39 \\
\hline $\mathrm{B}$ & $2.24 \times 10^{11}$ & $1.14 \times 10^{-10}$ & 0.87 & 1.40 \\
\hline $\mathrm{C}$ & $6.23 \times 10^{11}$ & $1.14 \times 10^{-8}$ & 0.88 & 1.06 \\
\hline
\end{tabular}

The ideality factor is in the range of 1 to 2 for all type of hydrogen sensor, which means the assumption of thermionic emission being the dominant current conduction mechanism is valid.

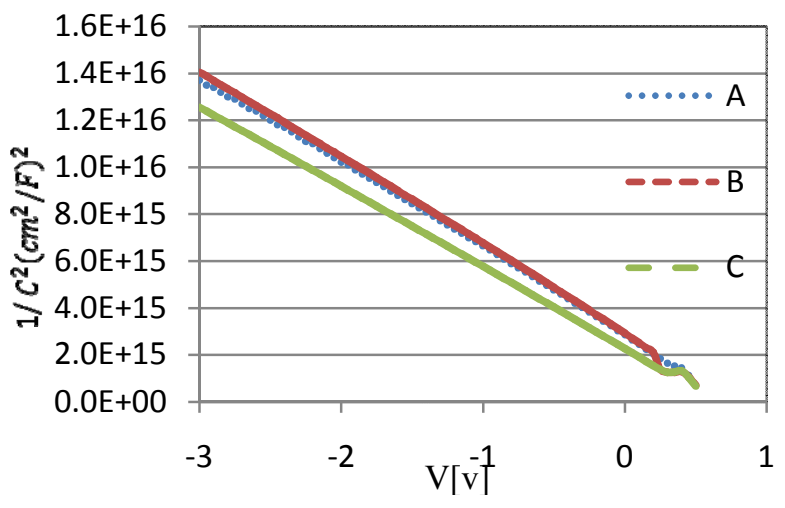

Figure 8: Capacitance-Voltage characteristics of PdNiSi Schottky barrier structures A, B, and $C$.

Fig 8 illustrates the dependence of $1 / C^{2}$ on applied voltage of Schottky contacts made on the medium resistivity silicon wafer, which is $1-2 \Omega$.cm by $\mathrm{PdNi}$ electrodeposition. The graph shows the predicted linear behaviour as described by Crowell [6]. Based on the slope and intercept, the various parameters are extracted. For our three structure samples, the parameters were scaled by the same contact area. The following table lists the doping concentration of the silicon substrates and the barrier heights of Schottky barrier based on the C-V measurement results.

Table 2: The $C$-V characteristic parameters of Schottky barrier, the Schottky contacts were made in the medium resistivity silicon wafer.

\begin{tabular}{|l|c|c|c|c|c|c|}
\hline Structure & $\begin{array}{c}N_{d} \\
(\mathrm{~A})\end{array}$ & $\begin{array}{c}V_{n} \\
(\mathrm{~A})\end{array}$ & $\begin{array}{c}V_{d o} \\
(\mathrm{~A})\end{array}$ & $\begin{array}{c}V_{i} \\
(\mathrm{~A})\end{array}$ & $\varphi_{B n}$ & $\eta$ \\
\hline $\mathrm{A}$ & $3.30 \times 10^{15}$ & 0.234 & 0.72 & 0.70 & 0.95 & 1.24 \\
\hline $\mathrm{B}$ & $3.14 \times 10^{15}$ & 0.236 & 0.75 & 0.72 & 0.98 & 1.41 \\
\hline $\mathrm{C}$ & $3.74 \times 10^{15}$ & 0.233 & 0.70 & 0.68 & 0.93 & 1.28 \\
\hline
\end{tabular}


Table 2 demonstrates that the fabricated devices and analysis were of a good standard, and shows that $\mathrm{C}-\mathrm{V}$ measurements is a method for determining doping concentration or resistivity which can compete with the four point probe method [7].

Fig 9 illustrates the I-V characteristics of the structure C Schottky barrier hydrogen sensor in the Nitrogen and hydrogen ambient. The sensor exhibits a low current in the Nitrogen ambient over the entire potential range. While the sensor under the hydrogen exposure, the modification of the $\mathrm{PdNi}$ work function changes the Schottky barrier at the interface leading to a current increase.

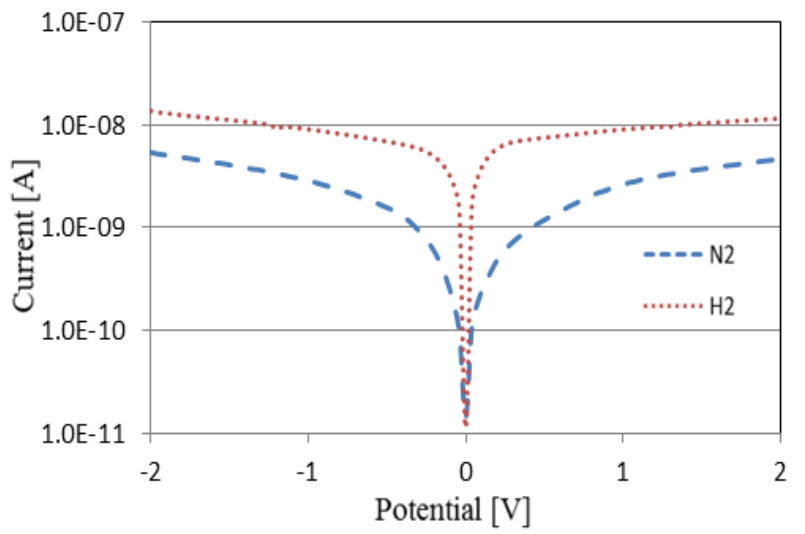

Figure 9: Electrical characteristic of back to back Schottky barrier hydrogen sensor (structure C) in Nitrogen ambient and in 5 mbar Hydrogen.

Fig 10 shows the time response of the devices after the exposure to 0.1 bar of hydrogen-nitrogen mixture. The response of the structure $\mathrm{C}$ has improved dramatically with a response time of less than a minute while structure $\mathrm{B}$ remains similar to structure A. This clearly indicates that hydrogen dissociation is not the limiting factor and that larger $\mathrm{Ni}$ concentration at the $\mathrm{Si}$ interface speeds up hydrogen capture.

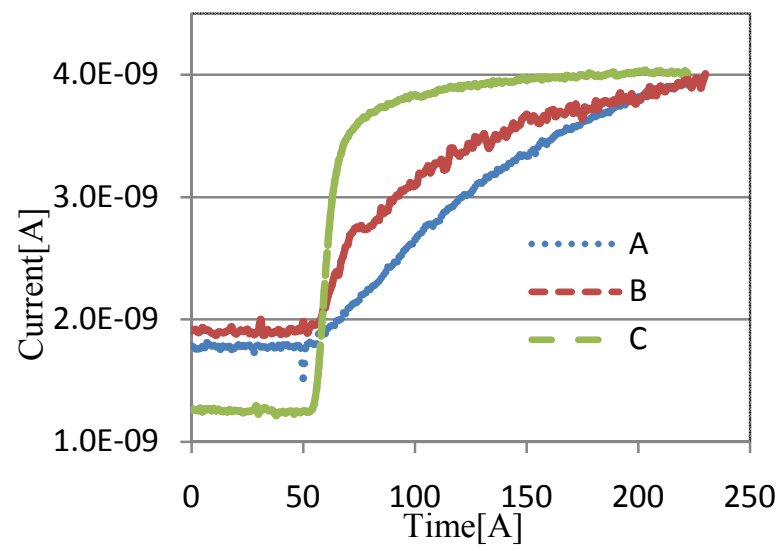

Figure 10: Time response of Hydrogen sensors A, B, and $C$ after the exposure to 5 mbar of Hydrogen at $50 \mathrm{~s}$.

\section{CONCLUSION}

We can extract the key parameters of these hydrogen sensors (such as Schottky barrier height, Si wafer resistivity, etc.) based on the dependence of $1 / C^{2}$ on applied voltage of Schottky contacts made on the medium resistivity silicon wafer $(1-2 \Omega . \mathrm{cm})$ and current (I)-Voltage (V) characteristics of PdNi-Si Schottky barrier hydrogen sensors. The fabrication and characterization of three different structure PdNi-Si Schottky barrier hydrogen sensors is presented. The new gradient structure sensor has a low idle current in Nitrogen ambient and exhibits a sensitive current change on exposure to 0.1 bar Hydrogen. The sensors shows selectivity responds to Hydrogen compared to Nitrogen. The response time of structure $\mathrm{C}$ has improved dramatically with a response time of around $15 \mathrm{~s}$ while structure B remains similar to structure A. This clearly indicates that hydrogen dissociation is not the limiting factor and that larger $\mathrm{Ni}$ concentration at the $\mathrm{Si}$ interface speeds up hydrogen capture and release.

\section{Acknowledgements}

The authors would like to acknowledge the Royal Society "Brian Mercer feasibility award" and support of INSU through their 2010 and 2011 CESSUR calls.

\section{REFERENCE}

[1].Hübert, T., Boon-Brett, L., Black, G., \& Banach, U. "Hydrogen sensors - a review." Sensors and Actuators B: Chemical, Vol 157, pp.329-352, 2011.

[2].William J. Buttner, Matthew B. Post, Robert Burgess, Carl Rivkin, "An overview of hydrogen safety sensors and requirements", International Journal of Hydrogen Energy, Vol. 36, Iss.3, pp.2462-2470, 2011.

[3].A.R. Usgaocar, C.H. de Groot, Cédric Boulart, Alain Castillo, Valérie Chavagnac, "Low power hydrogen sensors using electrodeposited PdNi-Si Schottky diodes", Sensors and Actuators B: Chemical, Vol 170, pp. 176181, 2012.

[4].Kiziroglou, Michail E. Zhukov, Alexander A. Abdelsalam, M. Li, Xiaoli, De Groot, Peter A J, Bartlett, N. de Groot, C.H."Electrodeposition of Ni-Si Schottky barriers," Magnetics Conference, 2005. INTERMAG Asia 2005. Digests of the IEEE International, 4-8 April 2005, pp.1197-1198

[5]. L. Dong, C.H. de Groot, A. Usgaocar, V. Chavagnac, "Electro-Deposited PdNi-Si Schottky Barrier Hydrogen Sensors with Improved Time Response", Procedia Engineering, Volume 47, pp 37-40, 2012.

[6]. C.R.Crowell, J.C.Sarace, and S.M.Sze, "TungstenSemiconductor Schottky barrier Diodes," Trans Met. Soc. AIME, Vol 23, pp478, 1965.

[7].W.F.Beadle, Quick Reference Manual for Semiconductor Engineer, Wiley, INC, 1985.

\section{CONTACT}

*L.Dong, tel: +44 (0)23 8059 3029; 1d4e09@ecs.soton.ac.uk 